Revista Arbitrada Interdisciplinaria de Ciencias de la Salud. SALUD Y VIDA

Volumen 5. Número 1. Año 5. Especial. 2021

Hecho el depósito de Ley: FA2016000010

ISSN: $2610-8038$

FUNDACIÓN KOINONIA (F.K)

Santa Ana de Coro, Venezuela.

Mildre Mercedes Vidal-del-Río; Alberto Sánchez-Garrido; José Fernando Trávez-Valencia;

Karla Alejandra Lascano-Álvarez

http://dx.doi.org/10.35381/s.v.v5i1.1614

\title{
Medicina ancestral y convencional de acuerdo con la cosmovisión de enfermedades culturales en Ecuador
}

\section{Ancestral and conventional medicine according to the cosmovision of cultural diseases in Ecuador}

\author{
Mildre Mercedes Vidal-del-Río \\ ua.mildrevidal@uniandes.edu.ec \\ Universidad Regional Autónoma de los Andes, Ambato \\ Ecuador \\ https://orcid.org/0000-0003-3496-5057 \\ Alberto Sánchez-Garrido \\ ua.albertosanchez@uniandes.edu.ec \\ Universidad Regional Autónoma de los Andes, Ambato \\ Ecuador \\ https://orcid.org/0000-0001-8317-2833 \\ José Fernando Trávez-Valencia \\ ma.joseftv27@uniandes.edu.ec \\ Universidad Regional Autónoma de los Andes, Ambato \\ Ecuador \\ https://orcid.org/0000-0002-2713-0353 \\ Karla Alejandra Lascano-Álvarez \\ ma.karlaala25@uniandes.edu.ec \\ Universidad Regional Autónoma de los Andes, Ambato \\ Ecuador \\ https://orcid.org/0000-0001-6659-6095
}

Recepción: 10 de agosto 2021

Revisado: 15 de septiembre 2021

Aprobación: 15 de noviembre 2021

Publicación: 01 de diciembre 2021 


\section{Sra. Editora:}

La medicina occidental, ha ido tomando gran importancia por su eficacia en el diagnóstico y tratamiento de patologías; sin embargo, Ecuador siendo un país multiétnico mantiene la medicina ancestral como una alternativa para muchos pueblos, principalmente indígenas quienes son los más creyentes en su efectividad, es necesario considerar que estas comunidades han convivido por siglos con la naturaleza, fomentando una armonía entre esta y el ser humano.

La aplicación de la medicina tradicional, no solamente se ve influido por la costumbre y tradición, además existen otros factores que determinan su uso en gran dimensión, un ejemplo es el ámbito socioeconómico, para estas personas les resulta una forma más económica sanar sus dolencias mediante el uso de plantas que el acudir a un centro médico que se ubica lejos de sus hogares y mucho menos el optar por una atención de un profesional. De tal manera, en nuestro país se han mantenido ambas medicinas vigentes y cumplen funciones tanto individuales como complementarias.

El tratamiento médico, se basa principalmente en asistir y contrarrestar la sintomatología que aqueja al paciente no a todos por igual, sino que prefieren analizar cada sistema por separado, evaluar su relación y posteriormente dar un diagnóstico e indicaciones; por el contrario, el manifestar todos estos síntomas frente a un sanador ancestral, este lo engloba en una sola patología "mal aire" o "espanto", no consideran simplemente un mal funcionamiento del organismo sino también la influencia del entorno frecuentado y estado anímico de quien lo sufre. Pese a las marcadas diferencias que existen entre ambos objetos de estudio, es necesario incentivar un incremento de aprendizaje de estas dos, especialmente en el personal de salud capacitado y darle importancia a esta medicina tradicional considerando que en Ecuador, la mayor parte de la población es mestiza, tiene raíces indígenas y confían en el uso de plantas medicinales así como en prácticas culturales, de esta manera, se fomenta una relación entre la medicina convencional y 
Revista Arbitrada Interdisciplinaria de Ciencias de la Salud. SALUD Y VIDA

Volumen 5. Número 1. Año 5. Especial. 2021

Hecho el depósito de Ley: FA2016000010

ISSN: 2610-8038

FUNDACIÓN KOINONIA (F.K)

Santa Ana de Coro, Venezuela.

Mildre Mercedes Vidal-del-Río; Alberto Sánchez-Garrido; José Fernando Trávez-Valencia;

Karla Alejandra Lascano-Álvarez

ancestral y se propone una divulgación más amplia de ambas para lograr una sinergia eficiente.

El mal aire y espanto son estados caracterizados por la pérdida del hálito en el paciente, el cuál presenta un estado febril y malestar general. Su atribución popular es a espíritus malignos (mal aire) o emociones repentinas (espanto) y por ende la rama preferida por los afectados ha sido la medicina ancestral. Sin embargo, a consulta de un médico general también se han presentado pacientes con síntomas propios del "mal aire" y de igual manera se le ha aplicado un tratamiento, pero ¿existe una relación entre la medicina ancestral y convencional en el tratamiento de mal aire y espanto?

Es este sentido, el objetivo de este estudio es describir la relación médico-clínica y ancestral sobre el mal aire para potenciar una cultura hibrida entre medicina científica y ancestral. Con ello se buscaría compensar las debilidades de una rama de la medicina con las fortalezas de otras para obtener la complementariedad y una mayor eficacia en el tratamiento.

\section{CONFLICTO DE INTERÉS}

Los autores declaran que no tienen conflicto de interés en la publicación del artículo.

\section{FINANCIAMIENTO}

No monetario.

\section{AGRADECIMIENTO}

A la Universidad Regional Autónoma de los Andes; por apoyar el desarrollo de la investigación. 


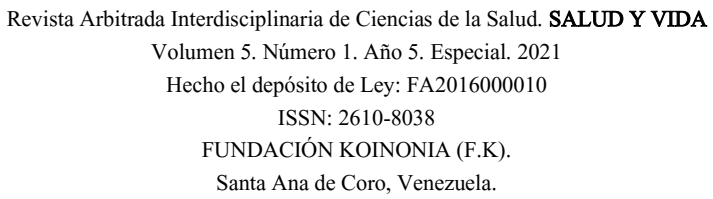

\section{REFERENCIAS}

1. Albán Pinto J, Vaca Cazares D. Análisis comparativo de: cosmovisión, y representaciones sociales del mal de aire en la ciudad de Pujilí (Hospital Rafael Ruiz) y Esmeraldas (Hospital Delfina Torres de Concha) con pacientes en zonas rurales, en relación con la medicina occidental en este tipo de enfermedades en sus dimensiones: médico, biológico, cultural de febrero 2018 a mayo 2018 [Comparative analysis of: cosmovision, and social representations of air sickness in the city of Pujilí (Hospital Rafael Ruiz) and Esmeraldas (Hospital Delfina Torres de Concha) with patients in rural areas, in relation to western medicine in this type of diseases in its dimensions: medical, biological, cultural from February 2018 to May 2018.]. [Internet]. Available from: http://repositorio.puce.edu.ec/handle/22000/15205

2. Yanchaguano Taco JM, Francisco Pérez Jl. Medicina convencional frente a medicina tradicional: preferencias de uso en una comunidad rural del Ecuador [Conventional versus traditional medicine: preferences of use in a rural community in Ecuador]. Conecta Libertad [Internet]. 2019; 3(2):44-5. Disponible en: http://revistaitsl.itslibertad.edu.ec/index.php/ITSL/article/view/82

3. Pereyra-Elías R, Fuentes Delgado D. Medicina Tradicional versus Medicina Científica ¿En verdad somos tan diferentes en lo esencial? [Traditional Medicine versus Scientific Medicine Are we really so different in the essentials?]. Acta méd. peruana [Internet]. 2012; 29(2): 62-63.

4. Casas Patarroyo C, Otero De La Hoz D, Mesa Melgarejo L, Carrillo A, Solano M. Prácticas de Medicina Tradicional y Complementaria en pacientes con neoplasias hematológicas [Traditional and Complementary Medicine practices on patients with hematologic neoplasic diseases]. Index Enferm [Internet]. 2017; 26(1-2): 2024.

5. Sousa I, Tesser C. Medicina Tradicional e Complementar no Brasil: inserção no Sistema Único de Saúde e integração com a atenção primaria [raditional and Complementary Medicine in Brazil: inclusion in the Brazilian Unified National Health System and integration with primary care]. Cadernos de Saúde Pública [online]. 2017; 33(1), e00150215. https://doi.org/10.1590/0102-311X00150215 
Revista Arbitrada Interdisciplinaria de Ciencias de la Salud. SALUD Y VIDA

Volumen 5. Número 1. Año 5. Especial. 2021

Hecho el depósito de Ley: FA2016000010

ISSN: $2610-8038$

FUNDACIÓN KOINONIA (F.K)

Santa Ana de Coro, Venezuela.

Mildre Mercedes Vidal-del-Río; Alberto Sánchez-Garrido; José Fernando Trávez-Valencia;

Karla Alejandra Lascano-Álvarez

6. Sousa IMC, Hortale VA, Bodstein RCA. Traditional Complementary and Integrative Medicine: challenges in constructing an evaluation model of care. Cien Saude Colet. 2018;23(10):3403-3412. doi:10.1590/1413-812320182310.23792016

2021 por los autores. Este artículo es de acceso abierto y distribuido según los términos y condiciones de la licencia Creative Commons Atribución-NoComercial-Compartirlgual 4.0 Internacional (CC BY-NC-SA 4.0) (https://creativecommons.org/licenses/by-nc-sa/4.0/). 